Vol. 7, n² | 2003

Varia

\title{
Frédéric Chauvaud, Les criminels du Poitou au XIX siècle, Les monstres, les désespérés et les voleurs
}

Geste Éditions, La Crèche 1999, 358 p., ISBN 291091927

\section{Martin Dinges}

\section{(2) OpenEdition}

\section{Journals}

Édition électronique

URL : https://journals.openedition.org/chs/584

DOI : $10.4000 /$ chs. 584

ISSN : 1663-4837

\section{Éditeur}

Librairie Droz

Édition imprimée

Date de publication : 1 décembre 2003

Pagination : 131-133

ISBN : 2-600-008990-3

ISSN : $1422-0857$

Référence électronique

Martin Dinges, «Frédéric Chauvaud, Les criminels du Poitou au XIXe siècle, Les monstres, les désespérés et les voleurs ", Crime, Histoire \& Sociétés / Crime, History \& Societies [En ligne], Vol. 7, n² I 2003, mis en ligne le 23 février 2009, consulté le 22 mars 2022. URL : http://journals.openedition.org/chs/584 ; DOl : https://doi.org/10.4000/chs.584

Ce document a été généré automatiquement le 22 mars 2022

(C) Droz 


\section{Frédéric Chauvaud, Les criminels du Poitou au XIX ${ }^{e}$ siècle, Les monstres, les désespérés et les voleurs}

Geste Éditions, La Crèche 1999, 358 p., ISBN 291091927

Martin Dinges

\section{RÉFÉRENCE}

Frédéric Chauvaud, Les criminels du Poitou au XIXe siècle, Les monstres, les désespérés et les voleurs, Geste Éditions, La Crèche 1999, 358 p., ISBN 291091927.

1 Ce livre comporte une thèse très nette, qu'il faut bien lire comme une critique fondamentale adressée à la recherche en histoire des criminalités des 30 dernières années en France : les statistiques de criminalité seraient lacunaires et elles «ne disent pas tout... même si elles s'avéraient totalement fiables et prolixes, elles ne fabriqueraient que des criminels désincarnés, abstraits, sans attaches locales, sans épaisseur et sans chair» (p. 10). Chauvaud juxtapose à ce constat le grand intérêt du $\mathrm{XIX}^{\mathrm{e}}$ siècle pour les personnages criminels, les circonstances du crime et à l'atmosphère qui entoure les méfaits. Il propose dès lors une autre approche, qui s'inscrit dans la perspective d'une « histoire anthropologique attentive aux évolutions lentes et souvent silencieuses, soucieuse de prendre en compte les sensibilités collectives longtemps inaperçues" (p. 11). Comme " méthode » il propose de multiplier les éclairages, croiser les sources et confronter les synthèses envoyées une fois par trimestre au ministère de la Justice avec les dossiers de procédure conservées à Niort et Poitiers.

Dans la première de trois parties admirablement équilibrées, Chauvaud note la place statistiquement tout à fait moyenne - du Poitou dans la "France criminelle ", non sans insister sur la valeur factice de cette donnée, qui ne refléterait que l'activité des tribunaux. Il présente la statistique morale comme enjeu de la politique régionale. C'est une des rares fois dans ce livre que la pertinence de l'espace comme catégorie pour une 
étude de criminalité paraît claire : ailleurs les jolies cartes de la distribution des crimes de sang, d'infanticides, d'incendies criminelles et d'autres délits sont comme un élément étranger au texte, qui ne s'y réfère point au delà des références à la taille des communes.

L'auteur décrit la construction du criminel par la presse et les tribunaux, ces derniers visant avant tout à établir la responsabilité du criminel. Suit un descriptif des formes d'interrogatoires, qui tairaient à de rares exceptions près «les aspects sociaux, les composantes culturelles et le caractère des inculpés » (69). Le travail des tribunaux est à juste titre caractérisé comme une machine à subsumer la trop grande variété de cas et de situations sous la définition légale d'un délit. Ce constat est réitéré pour deux vols tout à fait différents jugés néanmoins comme vols sur chemin public, donc aggravé sans que Chauvaud nous donne la durée des peines infligées par les juges, laquelle est normalement le moyen de respecter les différences des cas (212). En fait, il paraît que le reproche de l'auteur s'adresse plutôt aux historiens : selon lui, suivre le cheminement des statistiques judiciaires mettrait à plat des réalités multiformes. Cela ne devrait point être le travail de l'historien : sa tâche serait plutôt de reconstruire la diversité historique. Ainsi pour les techniques d'obtention de l'aveu ou le traitement des corps des détenus. Particulièrement intéressantes sont les pages sur l'interprétation des signes corporels des malfaiteurs comme révélateurs de leurs penchants criminels. Chauvaud donne ici une représentation statistique (exclusivement en \%!) des physionomies (nez et bouche) des voleurs telles qu'elles ont été observées par les cours d'assises.

4 La deuxième partie présente une approche narrative des criminels comme monstres froids ou chauds, une catégorisation point prévue dans les lois mais continuellement utilisée par les jurys et la presse. Sortir de toutes sortes de règles, agir sans motifs compréhensibles et abruptement sont quelques-unes des caractéristiques du monstre. L'argument de cette partie convainc, mais il aurait certainement gagné à une analyse quantitative et différentielle des discours de la presse et des jurés. Pour le vagabondage, l'inadaptation des catégories juridiques à des conditions concrètes est de nouveau soulignée. Chauvaud note la disparition du vagabondage, de l'infanticide et des révoltes de grain au cours du siècle - liant ici comme dans le livre entier l'histoire des criminalités à l'histoire économique et sociale de la région. Les fascinantes pages sur le vol et surtout sur le vol domestique incitent Chauvaud à remarquer que les dossiers sur les voleurs renseigneraient davantage sur ces personnages comme individus que sur leur environnement local. Pourquoi faut-il trancher - et ne pas en tirer des informations sur les deux aspects?

Dans une dernière partie sur le "désordre des émotions » Chauvaud montre bien comment les lieux peuvent renseigner - si ils sont bien enrichis par l'imaginaire; il analyse finement les manières dont les témoins comme les enquêteurs interprètent les indices du crime. Un bref historique des recherches sur les lieux et des manières de les représenter est un des multiples joyaux de ce livre. Une autre partie très innovatrice est l'analyse des trois formes de profit attendu du crime : agapes conviviales pour les hommes pauvres principalement; constitution de dot pour les femmes pauvres; sauver le petit commerce - grand instrument d'ascension sociale - par les (petits) fraudeurs; vouloir tout et maintenant pour les grands faussaires. Suit un descriptif des motifs du crime - de la haine ( $36 \%$ ) aux problèmes familiaux mal vécus (18\%). Pour les passions sexuelles, Chauvaud constate la disparition des viols collectifs après 1850 et souligne le 
rôle problématique des médecins experts, qui ne constatent un viol que sous condition que de très visibles blessures majeures au vagin des fillettes soient visibles. Ils s'insèrent ainsi dans une attitude d'occultation plutôt que de répression de ce crime.

6 En conclusion, c'est un livre riche de descriptions et d'analyses novatrices. Son approche est certainement innovante - pour l'histoire du crime au XIXe siècle et pour la France. Son approche étonne moins le lecteur moderniste ayant lu Yves Castan ou Arlette Farge, le premier étant encore davantage à la recherche de l'individu moyen de l'histoire des mentalités, la seconde soulignant tout l'enseignement à tirer des cas individuels. Chauvaud s'inscrit bien entendu dans une tendance de la recherche internationale. Il a globalement gagné son pari - difficile - de tenir l'équilibre entre la recherche des mentalités collectives et des situations individuelles abordées sous l'angle d'une anthropologie historique. Néanmoins l'utilisation de méthodes quantitatives - par exemple pour une analyse des discours des journaux et des jurés pourrait parfois renforcer son argument. Mais relativiser le quantitatif comme méthode utilisée trop exclusivement est dans doute nécessaire dans l'histoire des criminalités à la française - même si on sent bien le grand travail statistique en arrièreplan de ce livre important!

\section{AUTEURS}

\section{MARTIN DINGES}

Stuttgart, martin.dinges@igm-bosch.de 